\title{
Structure-Based Drug Design: Synergy of Structural Biology and Computational Methods
}

\author{
Leah L. Frye ${ }^{a}$ \\ aSchrödinger, Inc., 101 SW Main Street, Suite 1300, Portland, Oregon 97204, USA, \\ leah.frye@schrodinger.com
}

Structure-based drug discovery relies on the synergy between structural biology and computational methods. With access to a growing number of high quality protein x-ray, NMR, and cryo-EM structures, as well as advanced physics-based computational models, we can greatly accelerate all stages of the drug discovery process from target selection to clinical candidate. This presentation will discuss prospective, real world examples of the use of structurally-driven computational approaches to: 1) assess the druggability of potential ligand binding sites [1], 2) generate accurate binding poses [2], 3) identify novel lead compounds via virtual screening and core hopping $[2,3], 4)$ use the energetics of binding site waters to focus in on key regions for potency and selectivity optimization [4], 5) predict relative ligand binding affinities using free energy perturbation (FEP) methods to allow for the selection of optimal compounds for synthesis that address potency and/or selectivity issues [4]. In addition, machine learning coupled with extensive in silico simulation data allows for the efficient evaluation of millions, or even billions, of diverse compounds resulting in the need to purchase/synthesize only the most promising molecules.

\section{References}

[1] Halgren, T.A. (2009). J. Chem. Inf. Model., 49, 377-389.

[2] Murphy, R.B., Repasky, M.P., Greenwood, J.R., Tubert-Brohman, I., Jerome, S., Annabhimoju, R., Boyles, N.A., Schmitz, C.D., Abel, R., Farid, R., Friesner, R.A. (2016). J. Med. Chem., 59, 4364-4384. [3] Wang, L., Deng, Y., Wu, Y., Kim, B., LeBard, D.N., Wandschneider, D., Beachy, M., Friesner, R.A., Abel, R. (2017). J. Chem. Theory Comput., 13, 42-54.

[4] Abel, R., Mondal, S., Masse, C., Greenwood, J., Harriman, G., Ashwell, M.A., Bhat, S., Wester, R., Frye, L., Kapeller, R., Friesner, R.A. (2016). Curr. Opin. Struct. Biol., 43, 38-44. 